\title{
ALICE as a tool for programming at schools
}

\section{Ewa Graczyńska}

Institute of Mathematics, Opole University of Technology, Opole, Poland; e.graczynska@po.opole.pl

Received 7 November 2009, revised 24 November 2009, accepted 5 January 2010.

\begin{abstract}
We present some possible application of ALICE in the context of a possible attraction of pupils (especially girls) in early programming course. Our examples presented in the paper are fully explained on the base of [1].
\end{abstract}

Keywords: Interactive 3D Graphics;

Animation Authoring Tools

\section{INTRODUCTION}

Alice is an innovative 3D programming environment that makes it easy to create an animation for telling a story, playing an interactive game, or a video to share on the web. Alice is a teaching tool for introductory computing. It uses 3D graphics and a drag-and-drop interface to facilitate a more engaging, less frustrating first programming experience. In Alice's interactive interface, students drag and drop graphic tiles to create a program, where the instructions correspond to standard statements in a production oriented programming language, such as Java, $\mathrm{C}++$, and $\mathrm{C \#}$.

This information was found at www site: www.alice.org.

A free ALICE 2.2 programme may be found there, together with some other introductory materials.

Alice allows students to immediately see how their animation programs run, enabling them to easily understand the relationship between the programming statements and the behaviour of objects in their animation. By manipulating the objects in their virtual world, students gain experience with all the programming constructs typically taught in an introductory programming course.

Our aim is to point out that in our opinion this programme may be very useful as a tool for teaching in the Middle schools to attract pupils (especially girls) in: early programming, making short videos with MP3 music and adding some pieces of translations of that music, for example. Other possible applications at school is useful for learning foreign languages, especially English or German and other subjects as well. We intend to explain our ideas on three examples. These examples were examined on a group of pupils with a positive result.

It is not the purpose of this paper to publish statistical results on an application of the methods proposed but to show how a short presentation at school can be made in order to attract pupils in programming simple videos with their favourite MP3 music or with their own recordings of poems in their own or foreign language. In the third we use special effects. It is obvious that these three simple methods combined together presents a possibility of early introductory into programming in the Middle schools.

\section{AN EXAMPLE OF PRESENTING MP3 MUSIC WITH A DOMESTIC OR FOREIGN TEXT}

The author of [4] mentioned that: "Women are currently underrepresented in Computer Science". In the opinion of the author of [3] and many others: "middle school is a critical age, during which may girls turn away from scientific and mathematical pursuits, including Computer Science. By giving middle school girls a positive first programming experience, we may be able to increase girl's participation in Computer Science".

Also the opinion above has been made in USA, we have observed that the situation is quite similar in Poland and some other EU countries. In middle schools girls are less interested in Computer science as boys. As the result we observe that girls constitute a minority on Technical Faculties in our Technical Universities (including Opole University of Technology), on Informatics studies, while mathematical studies in Poland, surprisingly, are sometimes occupied by girls (for example at the University of Opole). Despite that fact, we have observed many times, that the scientific way for women, very often, is much more complicated and difficult as for men. However, this is not a topic of that article. Our goal is to concentrate on the particular problem of a possibility of increasing an interest of pupils in Computer Sciences in Middle schools, using the particular programme, namely ALICE. To achieve this goal, we present three possible ways of: 
1) implementing MP3 popular songs using ALICE,

2) implementing poems via ALICE,

3) using special effects and sounds.

We have observed that, programming short 1-3 minutes videos with MP3 music is very interesting for pupils, because one may learn programming and train its imagination at the same time, learn the text of the song plus English (or other language) by the way, Therefore we present here an example of a presentation of a piece of one popular song in Germany.

Our translation is a sample of possible texts which may appear in such video. First we present some pictures from the video then a possible code of such presentation, made with ALICE programme.

Example 1.

We present a photo made from a scene of our video: Party.mv by ALICE:

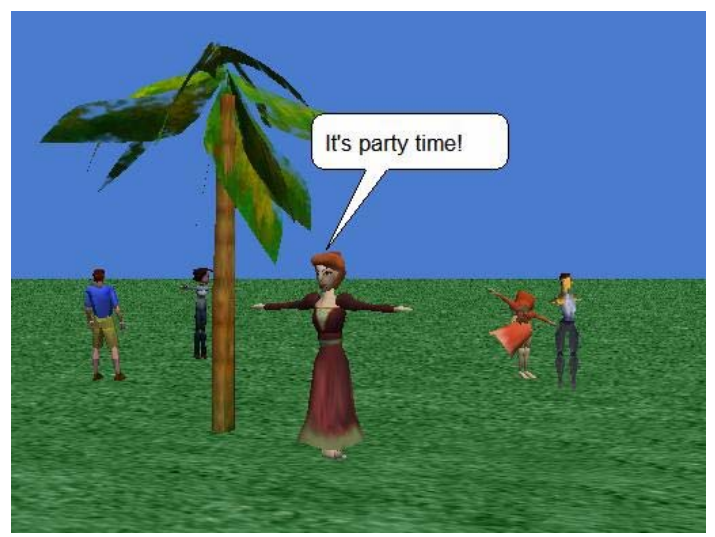

Diagram 1. A photo from video Party.mov.

Party's Code made for a 3 minutes video with MP3 music in German.

Party's Code

Created by: Ewa Graczyńska

World

Events

When the world starts

Do: world.music

When $\mathrm{A}$ is typed

Do: ground.dance1

Let move Any Object

\section{When 1 is typed}

Do: world.dialog1

\section{When 2 is typed}

Do: girl.dialog2
Methods

world.music( )

No variables

palmTree play sound world.10-AudioTrack $10(3: 44,896)$

duration $=\mathbf{1 5 0}$ seconds

world.dialog1 ( )

No variables

evilStepsister1 say It's party time! duration $=\mathbf{2}$ seconds

world.dialog3 ( )

No variables

randomGuy2 say You are super! duration $=\mathbf{2}$ seconds

Ground

Meyhods

ground.dance1 ( )

No variables

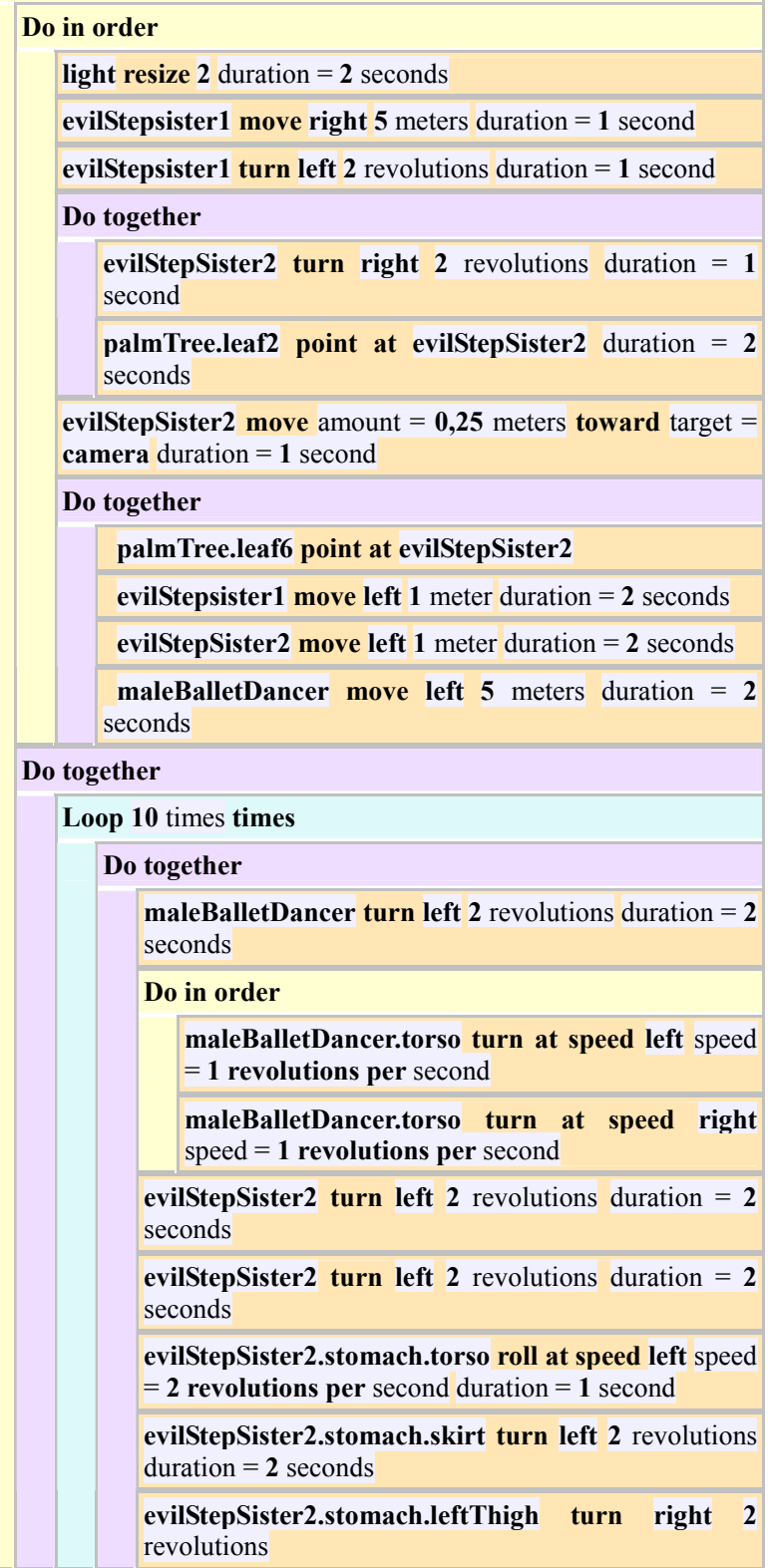


evilStepSister2 turn left 2 revolutions duration $=\mathbf{2}$ seconds

evilStepSister2.stomach.rightThigh turn left 2 revolutions

evilStepSister2.stomach.skirt roll at speed left speed $=2$ revolutions per second

randomGuy2 point at girl duration $=\mathbf{2}$ seconds style $=$ abruptly

Do together

maleBalletDancer turn left $\mathbf{2}$ revolutions duration $=\mathbf{2}$ seconds

Do in order

maleBalletDancer.torso turn at speed left speed $=1$ revolutions per second

maleBalletDancer.torso turn at speed right speed $=1$ revolutions per second

evilStepSister2 turn left 2 revolutions duration $=\mathbf{2}$ seconds

evilStepSister2 turn left 2 revolutions duration $=\mathbf{2}$ seconds

evilStepSister2.stomach.torso roll at speed left speed

$=\mathbf{2}$ revolutions per second duration $=\mathbf{1}$ second

evilStepSister2.stomach.leftThigh turn right 2 revolutions

evilStepSister2 turn left 2 revolutions duration $=\mathbf{2}$ seconds

evilStepSister2.stomach.rightThigh turn left 2 revolutions

evilStepSister2.stomach.skirt roll at speed left speed $=2$ revolutions per second

randomGuy 2 point at girl duration $=\mathbf{2}$ seconds style $=$ abruptly

Loop 10 times times

\section{Do together}

camera move backward 1 meter duration $=\mathbf{2}$ seconds girl stand up duration $=1$ second style $=$ gently girl turn left $\mathbf{0 , 2 5}$ revolutions duration $=\mathbf{2}$ seconds girl move left 1 meter duration $=\mathbf{2}$ seconds girl turn right 2 revolutions duration $=1$ second girl move forward 1 meter duration $=\mathbf{2}$ seconds girl turn at speed left speed $=2$ revolutions per second

evilStepsister1.hips orient to palmTree duration $=\mathbf{2}$ seconds

Do together

girl.body.leftArm turn left 2 revolutions

girl.body.rightArm turn right 2 revolutions

Do in order

girl.body.neck turn left 0,25 revolutions girl.body.neck turn right 0,5 revolutions girl.body.neck turn left 0,25 revolutions

girlleftLeg lowerLeg orient
girl.body.leftArm

evilStepsister1.hips.skirt turn to face girl.body evilStepsister1.hips.leftThigh.leftCalf turn left 2 revolutions

evilStepsister1.hips.rightThigh.rightCalf turn right 2 revolutions

Loop 2 times times

light turn left 1 revolution duration $=\mathbf{2}$ seconds style $=$ gently

camera get a good look at randomGuy2 asSeenBy $=$ evilStepsister1

randomGuy 2 orient to camera duration $=2$ seconds

randomGuy 2 turn right 0,5 revolutions duration $=2$ seconds

randomGuy 2 turn left 2 revolutions duration $=\mathbf{2}$ seconds

randomGuy2 orient to camera duration $=\mathbf{2}$ seconds

randomGuy2 turn left 0,5 revolutions

randomGuy2.upperBody turn at speed left speed $=\mathbf{2}$ revolutions per second

randomGuy2.leftLeg.knee turn at speed left speed $=\mathbf{2}$ revolutions per second

randomGuy2.rightLeg turn at speed right speed $=2$ revolutions per second

randomGuy2.leftLeg.knee turn right 2 revolutions

camera get a good look at girl duration $=\mathbf{2}$ seconds style $=$ abruptly

girl move amount $=1$ meter toward target $=$ camera duration $=$ 2 seconds

girl orient to camera duration $=\mathbf{2}$ seconds style $=$ abruptly

Do in order

light orient to girl duration $=\mathbf{2}$ seconds

girl orient to camera duration $=\mathbf{2}$ seconds

Loop 5 times times

girl.leftLeg turn right 2 revolutions duration $=1$ second

girl.rightLeg turn right 2 revolutions duration $=1 \mathrm{sec}-$ ond

girl.body turn right 0,25 revolutions duration $=\mathbf{1}$ second girl.body turn right 0,5 revolutions duration $=1$ second girl.body turn left 0,25 revolutions duration $=1$ second girl.body.neck turn left $\mathbf{0 , 2 5}$ revolutions duration $=\mathbf{1}$ second

girl.body.neck turn right 0,5 revolutions duration $=1$ second

girl.leftLeg orient to camera duration $=\mathbf{2}$ seconds style $=$ abruptly

girl.rightLeg orient to camera duration $=\mathbf{2}$ seconds style = abruptly

girl.rightLeg.lowerLeg turn right 2 revolutions duration $=\mathbf{1}$ second

girl.leftLeg.lowerLeg roll left 2 revolutions duration $=\mathbf{1}$ second

girl.body.leftArm roll left 2 revolutions duration $=\mathbf{1}$ second 
girl.body.neck.head orient to camera duration $=\mathbf{2}$ seconds style $=$ gently

girl.body.leftArm.forearm roll left 2 revolutions duration $=1$ second

girl.rightLeg roll right 2 revolutions duration $=\mathbf{1}$ second

girl.body.rightArm.forearm roll right 2 revolutions duration $=\mathbf{1}$ second

girl.body.neck turn left $\mathbf{0 , 2 5}$ revolutions duration $=\mathbf{1}$ second

girl turn left 2 revolutions duration $=\mathbf{1}$ second style = abruptly

light orient to girl

girl orient to camera duration $=1$ second style $=$ gently

Do together

randomGuy 2 stand up duration $=1$ second style $=$ gently

camera get a $\operatorname{good}$ look at randomGuy2 duration $=1$ second

Do together

camera get a good look at randomGuy2 duration $=\mathbf{2}$ seconds

randomGuy 2 point at girl duration $=\mathbf{2}$ seconds

camera get a good look at girl duration $=\mathbf{2}$ seconds

light orient to girl duration $=\mathbf{2}$ seconds

camera get a good look at girl.body duration $=\mathbf{2}$ seconds

light orient to girl.body duration $=\mathbf{2}$ seconds style $=$ gently

girl orient to camera duration $=\mathbf{2}$ seconds style $=$ gently

Do together

camera get a $\operatorname{good} \operatorname{look}$ at randomGuy2 duration $=\mathbf{2}$ seconds

\section{Do in order}

randomGuy2 say Hey! duration $=\mathbf{2}$ seconds

randomGuy2 say You are super! duration $=2$ seconds

evilStepSister2

Methods

evilStepSister2.dialog2 ( )

No variables

girl say We are dnacing and dancing. duration $=\mathbf{2}$ seconds

girl

Methods

girl.dialog2 ( )

No variables

girl say We are dancing! duration $=\mathbf{2}$ seconds

Diagram 2. Party's Code

We explain that in the code, MP3 music was arranged to play for 230 seconds. This time may be changed accordingly. "Sounds" are presented in ALICE as "seldom used properties". However, our experience led us to the conclusion that this kind of attracting pupils in making videos with ALICE work perfectly and gives many positive results.

\section{AN EXAMPLE OF PRESENTING OWN RECORDING}

\section{Example 2.}

Video Plato.mov may be considered for teachers in middle schools as another possibility to attract pupils in combining programming videos using ALICE and adding own sound, for example singing a song. This exercise in fact is quite difficult for beginners, as one should record its own graphical presentation of a poem (or a song) together with the voice suitably implemented. As our group of girls have practiced that to make a good recording via ALICE of a short poem or a song needs sometimes many tries. During these multiple repetitions, girls usually were able to learn to recite the poem which gave them additional advantage. Especially if the poem or a song was in a foreign language. Expressing the ides of a poem as a video was another positive experience which gave pupils a possibility to express more fully their emotions via colourful visualization. For some of them it was really a great pleasure to express thoughts in an animation powered with a piece of music or own declamation.

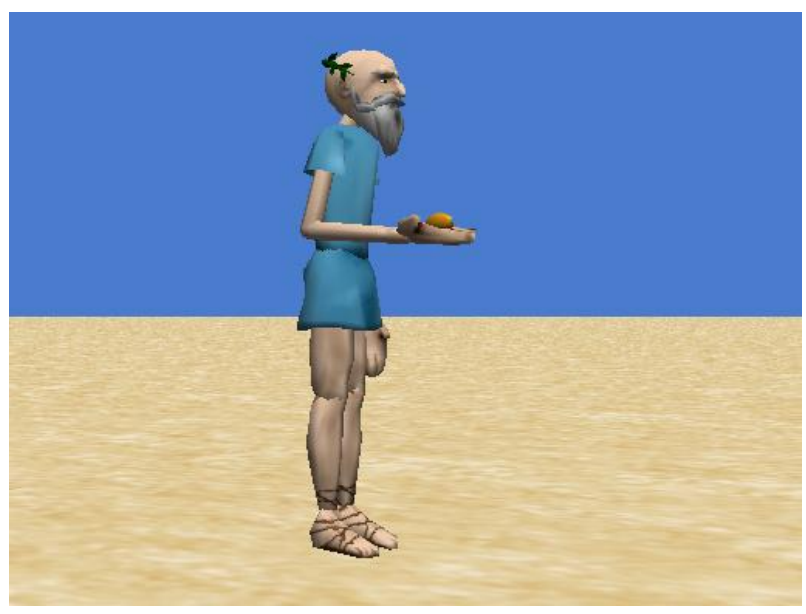

Diagram 3. A photo from video Plato.mov.

Plato's CodePlato's Code

Created by Ewa Graczyńska

World

Events

\section{When 1 is typed}

Do: world.metoda1

When 2 is typed 
Do: plato.metoda2

\section{When 3 is typed \\ Do: plato.metoda3}

Methods

\section{world.metoda1 ( )}

No variables

plato resize 2

plato

Methods

plato.metoda2 ( )

No variables

plato play sound plato.plato1 $(0: 04,650)$ duration $=$ Full Length

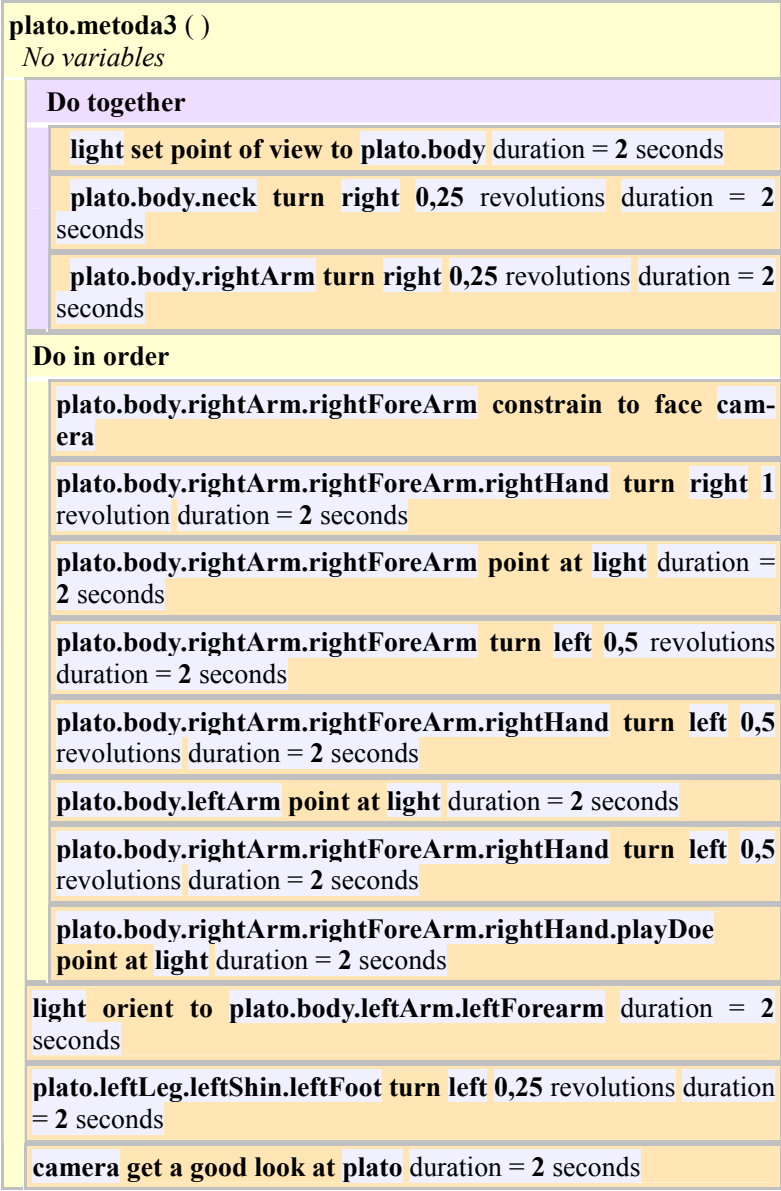

Diagram 4. Plato's Code

\section{AN EXAMPLE OF PRESENTING SPECIAL EFFECTS}

We studied monograph [1] to find some more possibilities for examples.
Example 3.

It presents a method of combining implementing sounds with special effects, as fire animations, to attract the presentation, based on Example 1 of [1], p. 307 where a class level variable timeLeft has been created to illustrate how a block of a possible game instructions can be controlled by a timer.

TIMER1's Code

Created by: Ewa Graczyńska

World

Events

\section{When the world starts}

Do: World.playGame

\section{When the world starts}

Do: World.my first method

\section{When the world starts}

Do: chicken.metoda1

\section{Let move Any Object}

\section{When $\mathrm{C}$ is typed}

Do: cloud.claud1

\section{When $\mathrm{F}$ is typed}

Do: fire.fire1

\section{When $\mathrm{G}$ is typed}

Do: fire.spin like crazy

\section{When the world starts}

Do: chicken.metoda2

\section{When $\mathrm{H}$ is typed}

Do: fireAnimation.fire2

Methods

World.my first method ( )

No variables

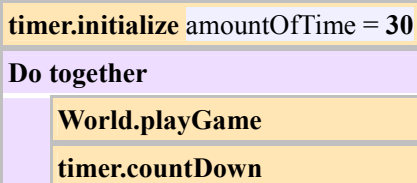

World.countdown ( )

No variables 


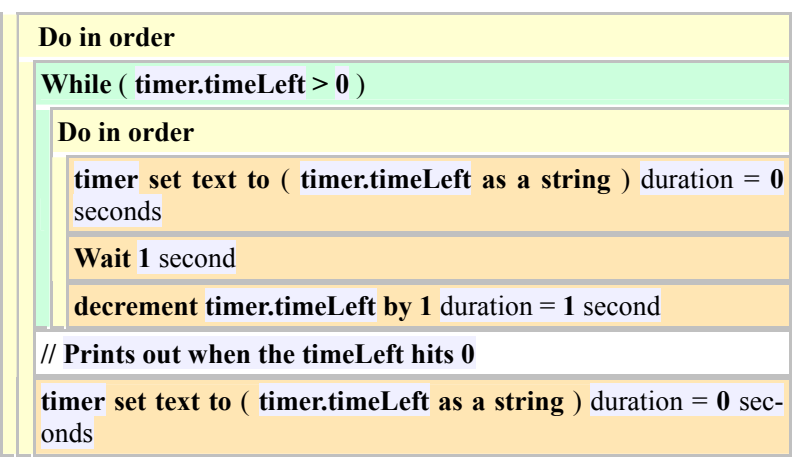

\begin{tabular}{|l|} 
World.game () \\
No variables \\
\begin{tabular}{|l|}
\hline Do in order \\
chicken play sound World.doorbell $(\mathbf{0 : 0 2 , 6 0 2 )}$ \\
\hline chicken play sound World.splash $(\mathbf{0 : 0 0 , 7 3 4 )}$ \\
chicken move amount $=\mathbf{2}$ meters toward target $=$ timer \\
\hline timer turn at speed right speed $=\mathbf{1}$ revolutions per second \\
\hline timer turn to face World \\
\hline
\end{tabular}
\end{tabular}

World.playGame ( )
No variables
\begin{tabular}{|l|} 
Do in order \\
While ( ( timer.howMuchtimeLeft $)>\mathbf{0})$ \\
chicken turn left $\mathbf{2}$ revolutions duration = $\mathbf{1}$ second \\
Loop 10 times times \\
chicken turn left 1 revolution duration = 1 second \\
Wait 1 second \\
chicken turn right 1 revolution duration $=\mathbf{1}$ second \\
\hline
\end{tabular}

Diagram 5. Timerl'st Code.

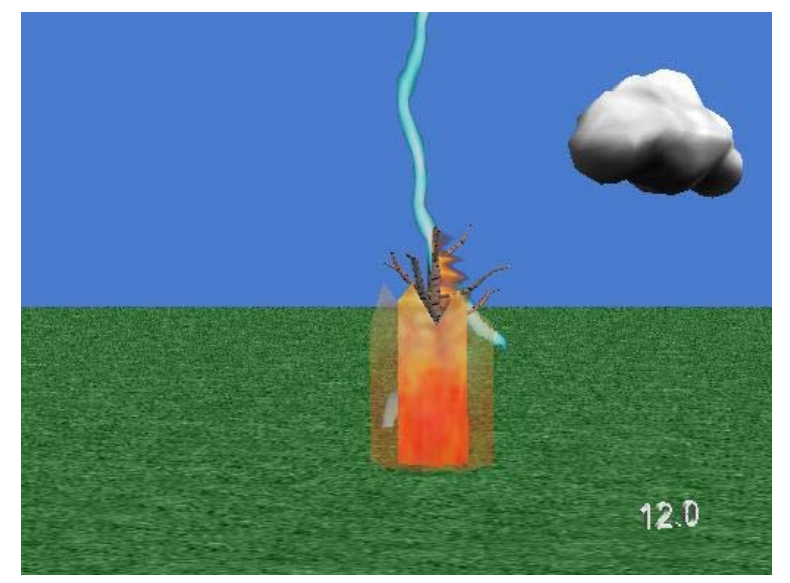

Diagram 6. A photo from video Timer1.mov.
We used instructions in Example 1 on p. 307 of [1] to create a timer that counts down in seconds. This exercise was exiting for pupils, as they used some new animations from the $\mathrm{CD}$ attached to the monograph [1]. Some pupils were very satisfied with that kind of programming as an early exercise before programming a more complicated game. During our short course of programming in Alice with a use of [1], in the group, the growth of an interest in programming games and also several other subjects was noted. Among them there were foreign languages, especially those in which the MP3 music was listened (English or German) or in which the poems were declaimed. A possibility of a computer visualization of ideas or some natural phenomenon was highly appreciated by the pupils as an additional effect of their experiments.

\section{ACKNOWLEDGEMENTS}

The author is very grateful to the referee for her/his valuable comments during the preparation of this paper. Due thanks are expressed to Wanda Dann for her early help to the author with Alice.

\section{REFERENCES}

[1] Cooper, S., Dann, W. and Pausch, R. (2009) Learning to program with ALICE, Pearson International Edition, Second Edition, Prentice Hall, London.

[2] Cooper, S., Dann, W. and Pausch, R. (2003) Teaching Objects-First in Introductory Computer Science. http://www.alice.org/publications/pubs/TeachingObjectsfirstInIntroductoryComputerScience.pdf.

[3] Kelleher, C. and Pausch, R. (2006) Lessons learned from designing a programming system to support middle school girls creating animated stories. IEEE Symposium on Visual Languages and Human-Centric Computing.

[4] Vegso, J., CRA Taulbee Trends: Female Students \& Faculty, http://www.cra.org/info/taulbee/women.html. Three files: Party.mov and Plato.mov and Timer1.mov are available at: www.egracz.po.opole.pl. 